\title{
Characterization of Secondary Electron Collection for Energy Recovery from High Energy Ions with a Magnetic Field*)
}

\author{
Shota HAGIHARA, Takayuki WADA, Satoshi NAKAMOTO, Hiromasa TAKENO, \\ Yasuyoshi YASAKA, Yuichi FURUYAMA ${ }^{1)}$ and Akira TANIIKE ${ }^{1)}$ \\ Electrical and Electronic Engineering, Kobe University, Kobe 657-8501, Japan \\ ${ }^{1)}$ Graduate School of Maritime Sciences, Kobe University, Kobe 658-0022, Japan
}

(Received 25 November 2014 / Accepted 6 February 2015)

\begin{abstract}
A traveling wave direct energy converter (TWDEC) is expected to be used as an energy recovery device for fast protons produced during the $\mathrm{D}-{ }^{3} \mathrm{He}$ nuclear fusion reaction. Some protons, however, are not fully decelerated and pass through the device. A secondary electron direct energy converter (SEDEC) was proposed as an additional device to recover the protons passing through a TWDEC. In our previous study, magnetic field was applied for efficient secondary electron (SE) collection, but the SEs were reflected close to the collector due to the magnetic mirror effect and the collection was degraded. Herein, a new arrangement of magnets is proposed to be set away from the collector, and experiments in various conditions are performed. An appropriate arrangement away from the collector resulted in the improvement of SE collection.
\end{abstract}

(C) 2015 The Japan Society of Plasma Science and Nuclear Fusion Research

Keywords: advanced fusion, direct energy conversion, secondary electron, magnetic mirror

DOI: $10.1585 /$ pfr. 10.3405025

\section{Introduction}

The $\mathrm{D}-{ }^{3} \mathrm{He}$ nuclear fusion reaction scheme has been considered to be an alternative nuclear fusion energy source for the future generations because neutrons are not produced in this reaction. Furthermore, most of the energy is released as the kinetic energy of charged particles, thus direct energy conversion (DEC) can be achieved. Consequently, an energy recovery device for protons that makes use of a traveling wave direct energy converter (TWDEC) was proposed [1]. However, it was discovered that some protons were not decelerated, which limited the efficiency of the device. In order to recover the protons passing through the TWDEC, an additional new devicea secondary electron direct energy converter (SEDEC) was proposed to be introduced in the downstream of the TWDEC [2]. As shown in the left part of Fig. 1, secondary electrons (SEs) are emitted when high-energy protons are incident on a metal foil, and the protons can penetrate the foil electrode and reach the next metal foil aligned in the direction of the proton beam. SEs are trapped by appropriately biased collectors and their energies are recovered. In this way the energy from the protons is indirectly recovered by collecting the SEs.

From the initial measurements, many SEs seemed not to arrive at the electron collectors placed around the electrodes but to arrive at the anteroposterior electrodes [2]. In order to guide the SEs to the collectors, the use of a magnetic field perpendicular to the proton beam was proposed.

author'se-mail: takeno@eedept.kobe-u.ac.jp

*) This article is based on the presentation at the 24th International Toki Conference (ITC24).

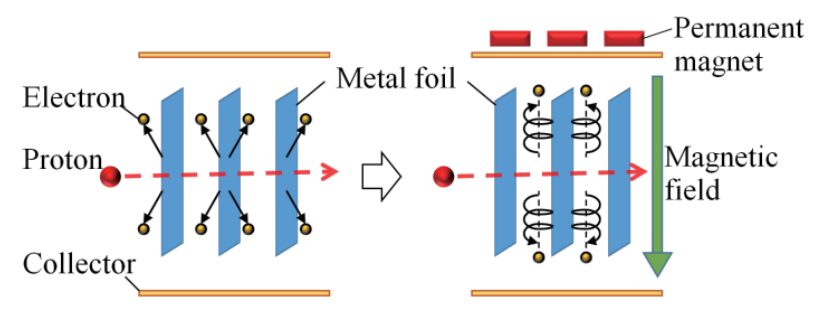

Fig. 1 Principle of an SEDEC with a magnetic field.

As shown in the right part of Fig. 1, a magnetic field is applied by permanent magnets to guide the SEs to the collectors. However, the number of SEs arriving at the anteroposterior electrodes was found to have decreased, whereas the number of SEs arriving at the collectors did not increase. The cause of this unusual result was explained using results from the orbit calculations, which showed that SEs were reflected near the collectors because of the magnetic mirror effect since the magnetic field was stronger close to the magnets [3]. A one-side arrangement of the magnets was tried and some improvement was found, but the amount of electrons collected was still less than that without magnets [4].

In this study, a further improvement of the scheme is considered. The reflection due to the magnetic mirror effect is determined by the mirror ratio between the collectors and the axis of the proton beam (generation point of $\mathrm{SE}$ ). This mirror ratio can be reduced by setting the magnets away from the axis to improve SE collection. The influence of the distance of the magnets from the axis on 
the SE collection is investigated experimentally.

In Sec. 2, the experimental equipment used is explained. The results of the experiments are shown in Sec. 3, including the discussions. The contents of the paper are summarized in Sec. 4.

\section{Experimental Equipment}

In the experiment, we used a tandem electrostatic accelerator (NEC 5SDH-2) as the high energy proton source. A $2.3 \mathrm{MeV}$ proton beam was used.

The fundamental structure of the SEDEC is shown in Fig. 2. Five foil electrodes and a back electrode are settled in a container. All the plates of the container are made of metal and are electrically connected. The whole of the container is used as an electron collector. The foil electrodes are made of aluminum. The first electrode (F1 in Fig. 2) is $18 \mu \mathrm{m}$, whereas the others (F2-F5 in Fig. 2) are each $5 \mu \mathrm{m}$ thick.

The normal to the foil electrode is inclined at $+15^{\circ}$ or $-15^{\circ}$ to the beam direction. When we assume that the collected SEs are only those directed toward the opposite side of the magnets, then the SEs are emitted from one side (front side for the case in Fig. 2) of the foil electrode because of this inclination. So, the arrangement in Fig. 2, with the normal inclined at $+15^{\circ}$, is referred to as a "front collection", whereas that with $-15^{\circ}$ is referred to as a "back collection". These two kinds of inclinations are examined for each magnet/electrode arrangement, which are described later.

The measurement circuit, by which the currentvoltage (I-V) characteristics for each electrode are measured, is also shown in Fig. 2. It should be noted that the meaning of "I-V characteristics" in this case is unusual: the voltage is applied to all the electrodes except the target (current measuring) electrode. For example in Fig. 2, F1 is the target electrode and F2-F5, the back electrode, and the container are biased to obtain the I-V characteristics of F1. When the bias voltage is negative, the target electrode is at a relatively high potential. So, the electrons flow into the target electrode, thus a negative current is observed. In the actual measurement, the ion source of the accelerator has a low frequency fluctuation and the measured current is also fluctuated. The beam current is monitored by a Faraday cup located upstream of the SEDEC. The target current is evaluated by proportional normalization to the beam current of $1 \mathrm{nA}$.

In the actual device used, various kinds of magnet/electrode arrangements are employed. Figure 3 shows two kinds of foil electrode arrangements. In pattern A, intervals between the foil electrodes, except for F1-F2, are wide, whereas they are narrow in pattern $B$.

The permanent magnet used is a rectangular parallelepiped-the sides are $35 \mathrm{~mm}$ by $20 \mathrm{~mm}$ by $5 \mathrm{~mm}$. The magnetization is in the direction of the thickness (the side of $5 \mathrm{~mm}$ ), and the surface magnetic field is $270 \mathrm{mT}$.

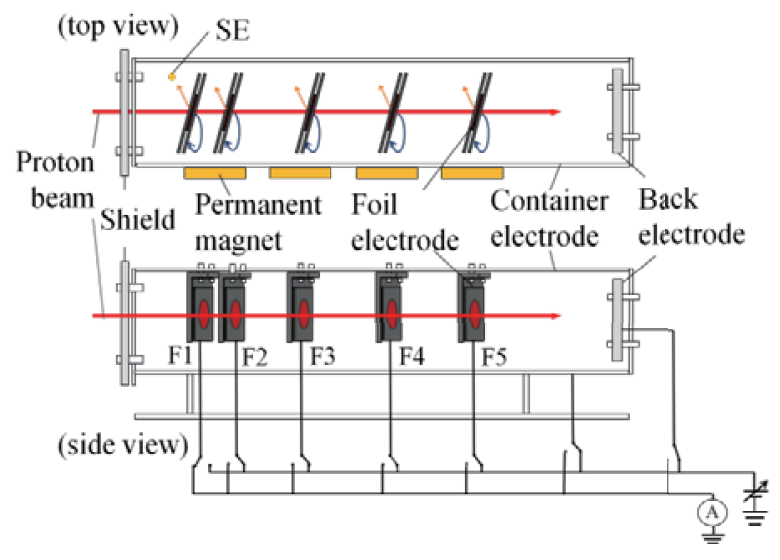

Fig. 2 Fundamental structure of the SEDEC.

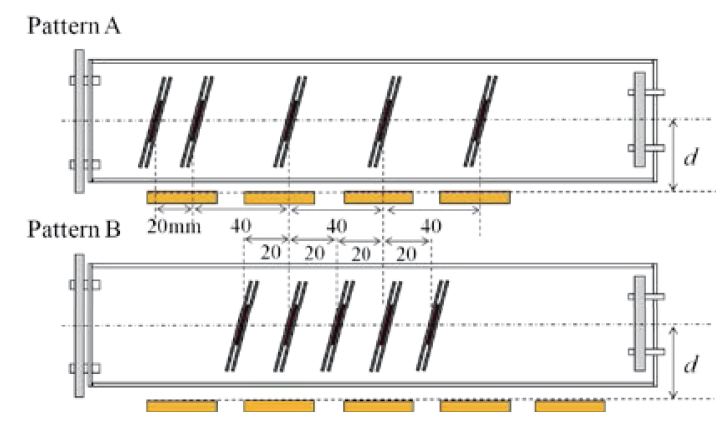

Fig. 3 Arrangements of foil electrodes.

Table 1 The number of magnets.

\begin{tabular}{|l|c|c|}
\hline & $26 \mathrm{~mm}$ & $86 \mathrm{~mm}, 146 \mathrm{~mm}$ \\
\hline Electrode & & 5 \\
\hline pattern A & 4 & 6 \\
\hline pattern B & 5 & \\
\hline
\end{tabular}

Three kinds of distances ( $d=26 \mathrm{~mm}, 86 \mathrm{~mm}$, and $146 \mathrm{~mm}$ ) perpendicular to the beam axis are taken, which are each defined by the distance between the axis and the surface of the magnet. The number of magnets is selected to cover the region occupied by the motions of the SEs, and is summarized in Table 1.

\section{Experimental Results and Discus- sion}

Figure 4 shows the I-V characteristics of electrodes F1-F5. The characteristics shown correspond to (a) without magnets, (b) front collection, and (c) back collection, for the pattern A electrode arrangement with $d=26 \mathrm{~mm}$. The variations due to magnetic field application are found in the first and the third quadrants. In the negative bias voltage regions, the negative current is reduced. This is because the electron motion in the axial direction is limited by the magnetic field, so the electron flow into the anteroposterior electrodes is reduced. As for the characteristics in the positive voltage region, the current is not sufficiently 


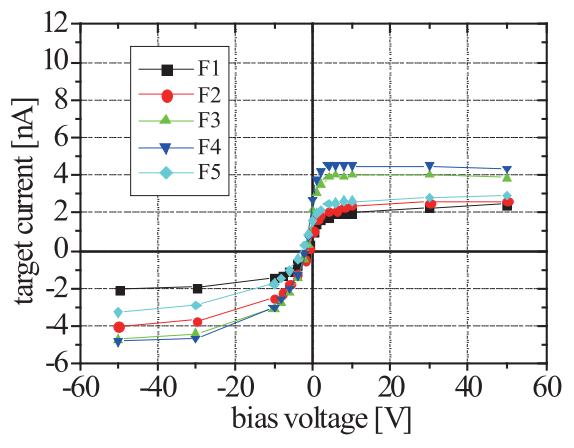

(a) without magnets

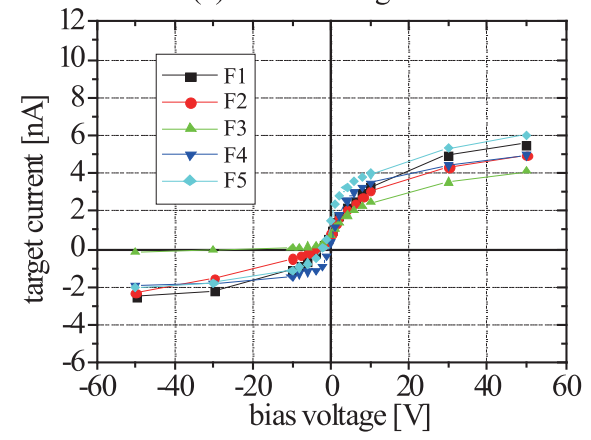

(b) front collection, pattern A, $d=26 \mathrm{~mm}$

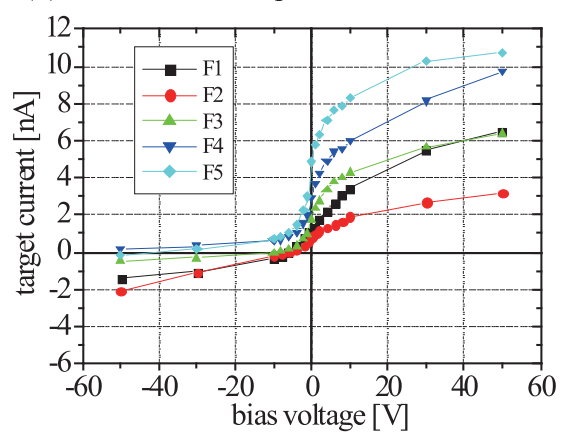

(c) back collection, pattern $\mathrm{A}, d=26 \mathrm{~mm}$

Fig. 4 I-V characteristics (dependence on magnetic field and front/back collection).

saturated by the applied magnetic field. Comparing (b) and (c), the amount of positive current for the back collection is more than that for the front collection except for F2. This may be because some field vectors around F2 have a component in the front direction, along which some SEs return to F2. In the case of $d=86 \mathrm{~mm}$, the average current is larger for the front collection. These phenomena are complex and the reason is not clear. The essential difference between the front and back collections will be evaluated at the end of the section.

Figure 5 shows the comparisons in terms of $d$ and the electrode pattern for the front collection. Figures 5 (a), (b), and (c) correspond to pattern A with $d=26 \mathrm{~mm}, 86 \mathrm{~mm}$, and $146 \mathrm{~mm}$, respectively. According to Figs. 5 (a), (b), and (c), the characteristics due to the applied magnetic field in Fig. 4 are gradually changed as $d$ increases, but with that for $d=146 \mathrm{~mm}$ being rather similar to that without magnets (Fig. 4 (a)). This is because the magnetic field strength, and thus the effect of motion limitation, is weak-

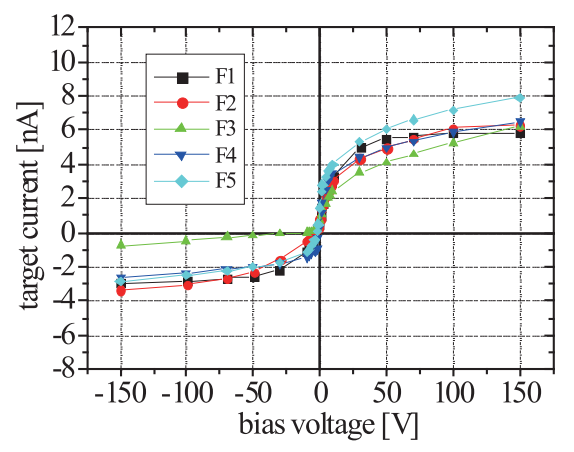

(a) front collection, pattern $\mathrm{A}, d=26 \mathrm{~mm}$

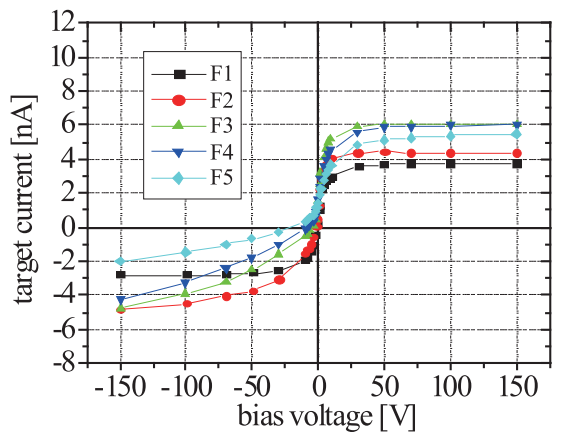

(b) front collection, pattern $\mathrm{A}, d=86 \mathrm{~mm}$

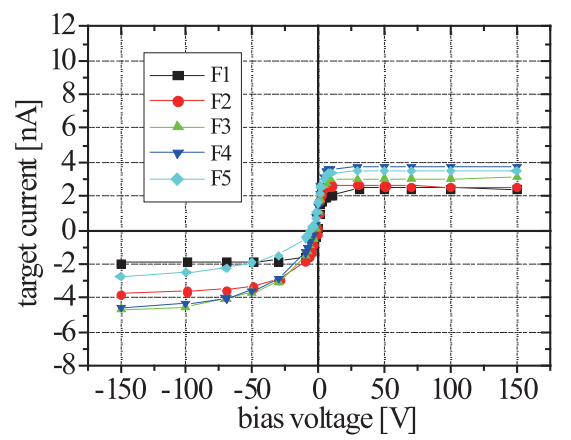

(c) front collection, pattern A, $d=146 \mathrm{~mm}$

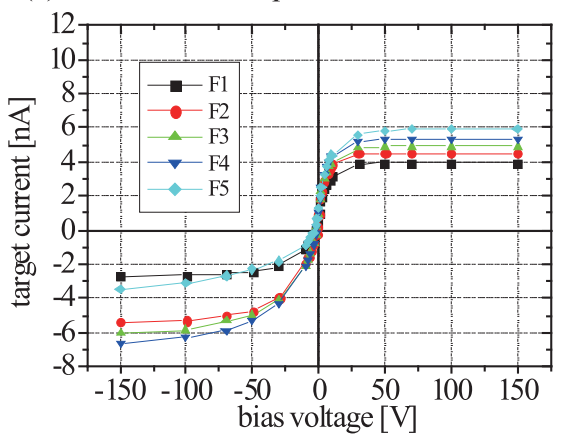

(d) front collection, pattern $\mathrm{B}, d=86 \mathrm{~mm}$

Fig. 5 I-V characteristics (dependence on $d$ and electrode arrangement).

ened as $d$ increases. As for Fig. 5 (d) that corresponds to pattern B with $d=86 \mathrm{~mm}$, it is similar to (b), but the amount of negative current in the negative voltage region is larger. This is because the interval between the electrodes for pattern B is smaller, so the SEs flow into the anteroposterior electrodes more easily.

The I-V characteristics are also measured for the 


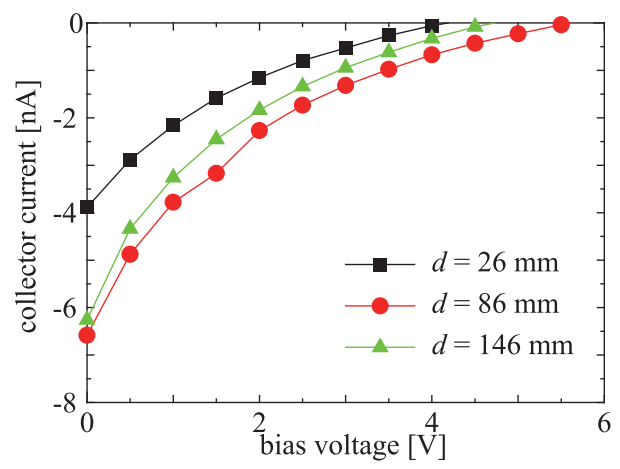

(a) pattern $\mathrm{A}$

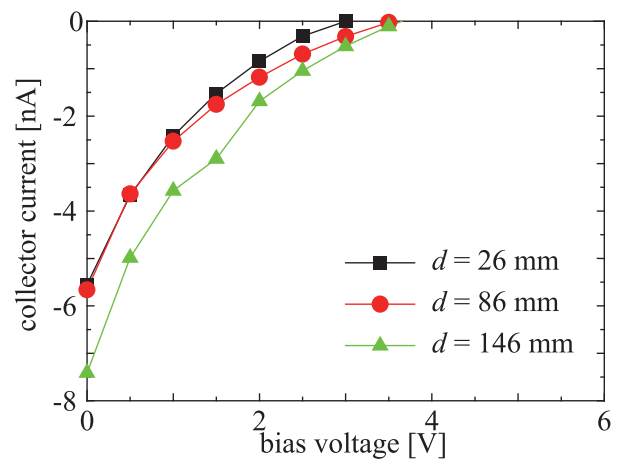

(b) pattern B

Fig. 6 I-V characteristics of the collector.

collector. The characteristics in the fourth quadrant are strongly related to the energy recovery of the SEs. The measured current in this region represents the amount of electrons arriving at the collector against the retarding field (more positive bias on the other electrodes than on the collector).

In Fig. 6, the I-V characteristics of the collector in the fourth quadrant for the front collection are shown, and (a) and (b) correspond to pattern A and B, respectively. According to Fig. 6 (a), the amount of negative current varies with $d$, and is maximized for $d=86 \mathrm{~mm}$. The highest voltage detecting current (HVDC) also varies and is also maximized $(5.5 \mathrm{~V})$ for $d=86 \mathrm{~mm}$. On the other hand, the amount of negative current is maximized for $d=146 \mathrm{~mm}$ in Fig. 6 (b) and variation of HVDC is small.

The amount of recovered power is evaluated by the product of the bias voltage and the collector current. In Fig. 7, the power-voltage characteristics are summarized for some cases: $d=86 \mathrm{~mm}$ for pattern A, $d=146 \mathrm{~mm}$ for pattern B, and the case without magnets. The highest powers are not so different for the above cases. The effective voltage region, however, is wide for pattern $\mathrm{A}$, and that for pattern $\mathrm{B}$ is almost the same as in that without magnets. This corresponds to the difference in I-V characteristics of the HVDC. The wider effective region has the advantage that multi-stage deceleration scheme [5] is applicable.

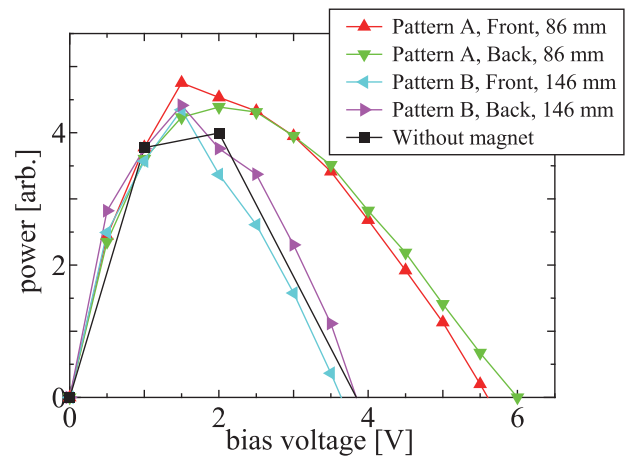

Fig. 7 Power-voltage characteristics.

The difference in $d$ of the HVDC is discussed briefly. If the limitation of the HVDC is due to the collision of the SEs with the anteroposterior electrodes during the cyclotron motions of electrons, an electron energy of $40 \mathrm{eV}$ is necessary for the Larmor radius of $20 \mathrm{~mm}$ since the typical field strength on the axis for $d=146 \mathrm{~mm}$ is about $1 \mathrm{mT}$. For $d=86 \mathrm{~mm}$, the mirror ratio at the collector to the axis is about 0.68 , thus an electron of $40 \mathrm{eV}$ on the axis translates its perpendicular energy of $13 \mathrm{eV}$ into a parallel energy. Based on this estimation, an HVDC of about $13 \mathrm{~V}$ can be expected, which is different from the observed value around $6 \mathrm{eV}$. The effect of the electric field should therefore be taken into account in the model of electron motion.

\section{Summary}

An improved scheme for an SEDEC with a magnetic field was examined. As the magnets were away from the beam axis, I-V characteristics of the foil electrodes gradually changed and approached those for the case without magnets. Considering an appropriate distance, the energy recovery was improved in terms of the effective voltage region. The spacing between the foil electrodes also affects the energy recovery so that SEs flow into the anteroposterior electrodes easily.

\section{Acknowledgment}

The authors acknowledge valuable discussions with Dr. J. Miyazawa.

[1] H. Momota, LA-11808-C, Los Alamos Natl. Lab., 8 (1990).

[2] D. Akashi et al., Trans. Fusion Sci. Tech. 63, 301 (2013).

[3] Y. Takeshita et al., 9th General Scientific Assembly of the Asia Plasma and Fusion Association in 2013, TP-36 (2013).

[4] S. Nakamoto et al., 10th Int. Conf. Open Magnetic Systems for Plasma Confinement, OS4-22 (2014).

[5] Y. Yasaka et al., Trans. Fusion Sci. Tech. 55, 1 (2009). 